Document downloaded from:

http://hdl.handle.net/10251/103789

This paper must be cited as:

Guardiola, C.; Climent, H.; Pla Moreno, B.; Reig, A. (2017). Optimal control as a method for diesel engine efficiency assessment including pressure and NOx constraints. Applied Thermal Engineering. 117:452-461. doi:10.1016/j.applthermaleng.2017.02.056

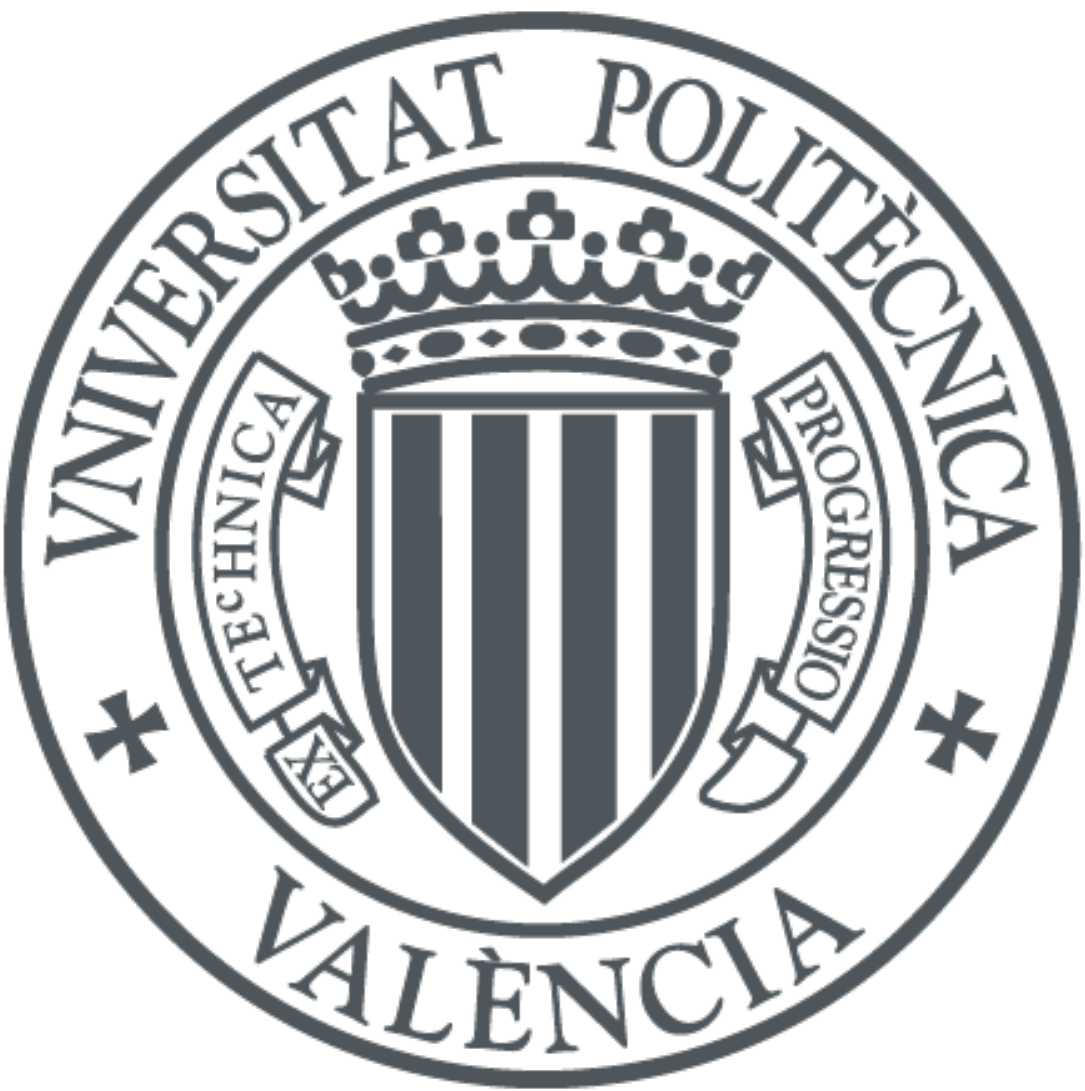

The final publication is available at

http://doi.org/10.1016/j.applthermaleng.2017.02.056

Copyright Elsevier

Additional Information 


\title{
Optimal Control as a method for Diesel engine efficiency assessment including pressure and $\mathrm{NO}_{\mathrm{x}}$ constraints
}

\author{
Carlos Guardiola, Héctor Climent, Benjamín Pla and Alberto Reig \\ CMT-Motores Térmicos, Universitat Politècnica de València \\ e-mail: \{carguaga, hcliment, benplamo, alreiber\}@mot.upv.es
}

\begin{abstract}
The present paper studies the optimal heat release law in a Diesel engine to maximise the indicated efficiency subject to different constraints, namely: maximum cylinder pressure, maximum cylinder pressure derivative, and $\mathrm{NO}_{\mathrm{x}}$ emission restrictions. With this objective, a simple but also representative model of the combustion process has been implemented. The model consists of a OD energy balance model aimed to provide the pressure and temperature evolutions in the high pressure loop of the engine thermodynamic cycle from the gas conditions at the intake valve closing and the heat release law. The gas pressure and temperature evolutions allow to compute the engine
\end{abstract} efficiency and $\mathrm{NO}_{\mathrm{x}}$ emissions. The comparison between model and experimental results shows that despite the model simplicity, it is able to reproduce the engine efficiency and $\mathrm{NO}_{\mathrm{x}}$ emissions. After the model identification and validation, the optimal control problem is posed and solved by means of Dynamic Programming (DP). Also, if only pressure constraints are considered, the paper proposes a solution that reduces the computation cost of the DP strategy in two orders of magnitude for the case being analysed. The solution provides a target heat release law to define injection strategies but also a more realistic maximum efficiency boundary than the ideal thermodynamic cycles usually employed to estimate the maximum engine efficiency.

Index Terms-Diesel Engine; Combustion Analysis; Optimal Control; Dynamic Programming

\section{INTRODUCTION}

$\mathbf{T}$ HE increasingly stricter emission regulations have forced an important evolution in the Diesel engine

Thanks are due to the Ministerio de Economía y Competitividad by its financial support through project $\mu$-Balance (TRA2013-41348-R) technology during the last 25 years. This evolution has led to noticeable reductions in the emission levels of such engines, nevertheless, some of those techniques have negative effects on engine efficiency and then the impact of the Diesel technology evolution on the reduction of fuel consumption has not been as high as desired. Accordingly, along decades, the main focus in engine design has been emissions. However, economical crisis, increasing social awareness about the global warming and limited oil resources have resulted in the demand of more efficient engines that both reduce fuel consumption (and then Carbon dioxide $\left(\mathrm{CO}_{2}\right)$ print) without penalties on performance and emissions. This demand has been also materialised in a growing regulatory pressure to reduce green house gases and particularly $\mathrm{CO}_{2}$. Under this scope, tools that help to analyse the tradeoff between fuel consumption and emissions are foreseen. Traditionally, energy balance [1]-[4] has been one of these techniques, aiming to study the mass and energy flows into and out of the different engine systems in order to identify possible undesirable energy sinks affecting efficiency. A more refined approach consists of using both the first and second Law of Thermodynamics in order to perform an exergy (available energy) balance that takes into account irreversibility in engine processes such as combustion [5], [6]. Previous approaches allow the identification of potential efficiency improvements by recovering part of the thermal energy loss, and particularly, the second one allows to find 
an upper bound for efficiency given engine characteristics and its operating conditions. However, despite dealing with the minimisation of energy losses, previous methods are not wellsuited to take into account other parameters with important impact on engine emissions and design. Another important issue of previous methods is that the complexity of the combustion process requires the assumption of an arbitrary law of heat release. In this sense, traditional thermodynamic processes such as constant volume, constant pressure and limited pressure combustions are assumed [5], or more sophisticated Wiebe functions are considered [6].

In the present paper, authors follow a third possible approach: Optimal Control (OC) recently employed by [7], [8] to find the heat release law (HRL) in an internal combustion engine that minimises fuel consumption with pressure and knock constraints. The advantages of the OC approach are twofold:

1) Restrictions related to non-energy based parameters can be taken into account. In this sense, the main contribution of the present work is that it provides a higher bound for the engine efficiency taking into account restrictions on maximum cylinder pressure, maximum cylinder pressure derivative and Nitrogen oxides $\left(\mathrm{NO}_{\mathbf{x}}\right)$ emissions. Moreover, the framework presented in this paper is completely general and other pollutant emissions (or criteria) can be also included if a suitable model exists.

2) As far as it is optimal, it provides the best possible combustion scenario for comparison. Instead of using arbitrary combustion laws such as constant volume, or Wiebe functions, the OC shows the HRL that minimises the defined cost function while strictly fulfilling the problem constraints.

In line with the previous paragraphs, the purpose of this paper is to obtain by means of OC, the HRL that minimises the fuel consumption at some defined operating conditions, taking into account different mechanical and performing restrictions, particularly: the maximum cylinder pressure should be bounded to avoid engine damage; the maximum pressure derivative in the combustion chamber should be also limited to avoid both mechanical issues and noise emissions; finally, provided the tradeoff between $\mathrm{NO}_{\mathrm{x}}$ and consumption in current Diesel engines, some boundary in the maximum $\mathrm{NO}_{\mathbf{x}}$ is to be included to fulfil with emission regulations. In this sense, the proposed approach will provide both a realistic minimum fuel consumption to compare how optimal is a given engine calibration and also a HRL pattern to follow in order to minimise the engine fuel consumption respecting the defined restrictions.

The paper is organised as follows: In section II, a zero dimensional model able to compute the indicated work and $\mathrm{NO}_{\mathbf{x}}$ emissions from the heat release law and the gas conditions at the intake valve closing is briefly described. The interested reader is referred to [9] where a complete description of the model can be found. After the model validation with experimental data in section III, the Optimal Control problem is formulated and the method used to solve it, Dynamic Programming (DP), is introduced in section IV. The results obtained are shown in section $\mathrm{V}$, where the effect of considering different constraints on the optimisation problem is assessed. Since one of the main drawbacks of the OC and particularly of DP is the computational cost, an explicit optimal HRL policy is derived that can reduce computational burden of numerically challenging DP for the case of pressure constraints. Finally, section VI outlines the main conclusions and contributions of the paper.

\section{ENGINE MODEL}

In the present paper, the compression, combustion and expansion processes of a Diesel combustion engine, i.e. be- 
tween Intake Valve Closing (IVC) and Exhaust Valve Opening (EVO), are modelled as a process in a closed system with a single substance whose properties change with the thermodynamic conditions according to the correlations from [10].

The combustion process is modelled as a heat addition to the system. Particularly, the heat addition is the control variable in the present formulation. On the other hand, the heat transferred to the cylinder walls is modelled as a function of the temperature difference between the gas and the cylinder walls with a version of the so called Woschni approach [11]. The considered system is modelled as a one-component (gas whose properties are a weighted average of the air, fuel and exhaust gas properties) homogeneous (gas phase) system, then its thermodynamic state is fully defined by 2 variables, hereinafter state variables. In the present paper, volume $(V)$ and pressure $(p)$ are chosen as state variables. This selection is justified by the fact that $V$ depends on the slider-crank mechanism, so its evolution, for a given engine geometry and operating speed, is independent of the system variables and can be considered as a disturbance in the control sense. Particularly, as far as the air loop is not included in the presented analysis, the mass of the system can be considered a priori known and $V$ can be replaced by the specific volume $v$. Regarding the selection of $p$ as a secondary state variable instead of the more usual selection of the temperature $(T)$ in thermodynamic problems, the reason is that it would make easier to include the maximum pressure constraints in the Optimal Control Problem (OCP). Accordingly, provided the volume during the thermodynamic cycle, the cylinder pressure evolution can be obtained by integration of the first law of thermodynamics applied to the closed system between the IVC and EVO:

$$
\mathrm{d} p / \mathrm{d} \alpha=\frac{\gamma-1}{v}\left(\delta q_{b} / \mathrm{d} \alpha+\delta q_{\text {walls }} / \mathrm{d} \alpha\right)-\frac{\gamma}{v} p \mathrm{~d} v / \mathrm{d} \alpha
$$

where the dependence of the variables on the crank angle $(\alpha)$ has been omitted for the sake of clarity, $v$ is the specific volume in the cylinder, $\gamma$ is the heat capacity ratio, $\delta q_{b}$ and $\delta q_{\text {walls }}$ represent the heat released during the combustion process and the heat transfer to the cylinder walls respectively. Note that equation (1) is obtained from the application of the First Law of Thermodynamics to a closed system, which involves that there is not mass exchange between the system and its surroundings. In the case at hand it means that since the injection process involves some mass exchange, the fuel mass injected should be neglected in front of the total mass admitted in the cylinder. As far as the stochiometric fuel to air ratio for the considered fuel is $1 / 14.5$ and taking into account the lean operation of the Diesel engine (below 0.6-0.7) the fuel only represents around a $4 \%$ of the total mass admitted by the cylinder, so this assumption can be done without introducing a significant error. The heat released during the combustion is computed as:

$$
\delta q_{b} / \mathrm{d} \alpha=m_{f} L H V u
$$

where $m_{f}$ is the fuel injected during the cycle, $L H V$ stands for the Lower Heating Value of the fuel and $u$, which is the control variable, represents the fuel burning rate, i.e. the derivative of the fuel mass fraction burnt respect to the crank angle.

Regarding the heat transfer to the cylinder walls, it is modelled with a Nusselt like correlation, particularly a variation of the Woschni's correlation proposed in [3] and [4].

$$
h=C B^{-0.2} p^{0.8} T-0.53 v_{g}^{0.8}
$$

where $C$ is a constant (in the case at hand 0.12 ), $B$ is the piston bore, $p$ and $T$ represent the pressure and temperature evolution in the combustion chamber and $v_{g}$ is the gas velocity obtained from the following correlation: 


$$
v_{g}=C_{W 1} c_{m}+C_{W 2} c_{u}+C_{2} \frac{V_{d} p_{I V C}}{V_{I V C} T_{I V C}}\left(p-p_{0}\right)
$$

where $c_{m}$ and $c_{u}$ are the mean piston speed and the tangential flow velocity due to swirl respectively, $p_{I V C}, V_{I V C}$ and $T_{I V C}$ are the cylinder pressure, volume and temperature at the intake valve closing, $V_{d}$ is the cylinder displacement, $p_{0}$ is the pressure evolution in motoring conditions and $C_{W 1}, C_{W 1}$ and $C_{2}$ are calibration constants.

The integration of equation (1) from the IVC to the EVO allows to compute the pressure evolution that finally can be used to calculate the indicated work. It is straightforward to compute the indicated efficiency or the indicated specific fuel consumption (ISFC) which is the function to minimise in the present study. Note that the ISFC minimisation is completely analogous to the indicated efficiency or indicated work maximisation.

Regarding the restrictions to be applied to the ISFC minimisation, those related with pressure, i.e. maximum cylinder pressure and maximum cylinder pressure derivative can be directly assessed from the cylinder pressure trace. Regarding the $\mathrm{NO}_{\mathbf{x}}$ constraint, an additional model to compute the $\mathrm{NO}_{\mathbf{x}}$ emissions associated to a given HRL should be used. There are several low computational cost models to predict $\mathrm{NO}_{\mathbf{x}}$ emissions in the literature, some of them rely on correlations that provide the $\mathrm{NO}_{\mathbf{x}}$ emitted depending on the operating conditions [12], [13], while others, more physically based, use the evolution of the thermodynamic variables during the cycle to compute the in-cycle evolution of the $\mathrm{NO}_{\mathbf{x}}$. In the present study, one of the last kind proposed by [9] has been used because of its good accuracy and the easy combination with the combustion model previously presented. According to the referred model, the thermal $\mathrm{NO}_{\mathbf{x}}$ production can be calculated as:

$$
\frac{\delta N O_{x}}{\mathrm{~d} \alpha}=k_{f} \frac{\delta q_{b}}{\mathrm{~d} \alpha} \exp \left(-k_{a c t} /\left(T_{a d}-k_{\Delta T}\right)\right)
$$

where $k_{f}$ represents the proportionality between the heat released and the amount of reactants passing through the flame front, $k_{a c t}$ is a constant to model the $\mathrm{NO}_{\mathbf{x}}$ reaction rate with an Arrhenius-like correlation depending on the temperature of the reactants, that at the present work is estimated as the adiabatic temperature $\left(T_{a d}\right)$ with some deviation represented by the calibration parameter $k_{\Delta T}$. A deep explanation of the physical background of the previous expression can be found in [9]. Note that the used model only takes into account $\mathrm{NO}_{\mathbf{x}}$ formation via the thermal mechanism, so despite being the more important in Diesel engines, some deviation between the model results and experimental $\mathrm{NO}_{\mathbf{x}}$ emissions can be observed, specially when premixed combustion plays an important role [9].

\section{EXPERIMENTAL FACILITY AND MODEL VALIDATION}

A single cylinder Direct Injection Compression Ignition (DICI) engine, whose main features are shown in table I, was employed for the present study. Despite the optimisation is applied to the model described in section II, the experimental facility is used to validate the model, and also to evaluate the engine performance in comparison with the optimal results provided by the $\mathrm{OC}$ approach. The single cylinder engine employed in the present paper is the result of an inhouse engine design process aimed to develop the minimum size DICI engine for automotive applications (specific power around $40 \mathrm{KW} /$ litre and $40 \%$ efficiency) with state of the art technology [14]. The resulting engine, with $150 \mathrm{~cm}^{3}$ displacement, is fully instrumented with temperature, pressure, mass flow and concentration sensors. For this particular study, the key signals recorded are the in-cylinder pressure signal and fuel consumption to compute the ISFC, the air mass flow and intake conditions to compute the gas conditions at the IVC 
and the $\mathrm{NO}_{\mathbf{x}}$ emissions to assess the emission limits.

\begin{tabular}{ll}
\hline Type & common rail DI \\
Number of cylinders & 1 \\
Bore/Stroke & 1.1 \\
Displacement & $150 \mathrm{~cm}^{3}$ \\
Compression ratio & $17: 1$ \\
Combustion chamber geometry & quiescent \\
Swirl number & 0 \\
Maximum cylinder pressure & $180 \mathrm{bar}$ \\
Number of injection nozzles & 6 \\
Nozzle diameter & $92 \mu \mathrm{m}$ \\
\hline
\end{tabular}

TABLE I: Engine main characteristics.

All the tests have been done in a single operating point defined by its engine speed (3000 rpm) and fuelling rate (14.7 mg/cycle). Table II shows a summary of the operating conditions. A parametric study modifying key engine variables such as the relative fuel to air ratio (FAR), injection pressure, injection timing and number of injections has been carried out according to the ranges shown in table II.

\begin{tabular}{ll}
\hline engine speed & $3000 \mathrm{rpm}$ \\
fuel injected & $14.7 \mathrm{mg}$ \\
air & $377 \mathrm{mg}$ \\
intake temperature & $35^{\circ} \mathrm{C}$ \\
FAR & {$[0.5,0.7]$} \\
Injection pressure & {$[1200,1400]$} \\
Maximum cylinder pressure & $160 \mathrm{bar}$ \\
Number of injections & {$[1,2]$} \\
\hline
\end{tabular}

TABLE II: Operating conditions.

Figure 1 shows a comparison between measured and modelled ISFC and $\mathrm{NO}_{\mathbf{x}}$ emissions. The agreement between the measured and modelled ISFC for the set of tested conditions is between the $\pm 2.5 \%$ error, and it increases to $\pm 10 \%$ in the case of $\mathrm{NO}_{\mathbf{x}}$ emissions. While the model uncertainty in terms of ISFC seems adequate, the error in $\mathrm{NO}_{\mathbf{x}}$ may appear excessive. Nevertheless, Arrègle et al. [13] point out that a variation of $\pm 1 \%$ in the estimation of each of the parameters that affect $\mathrm{NO}_{\mathbf{x}}$ translates into an error up to $\pm 33 \%$ in final $\mathrm{NO}_{\mathrm{x}}$ prediction. This extremely high sensitivity is a direct consequence of the involved physic-chemical mechanisms, and
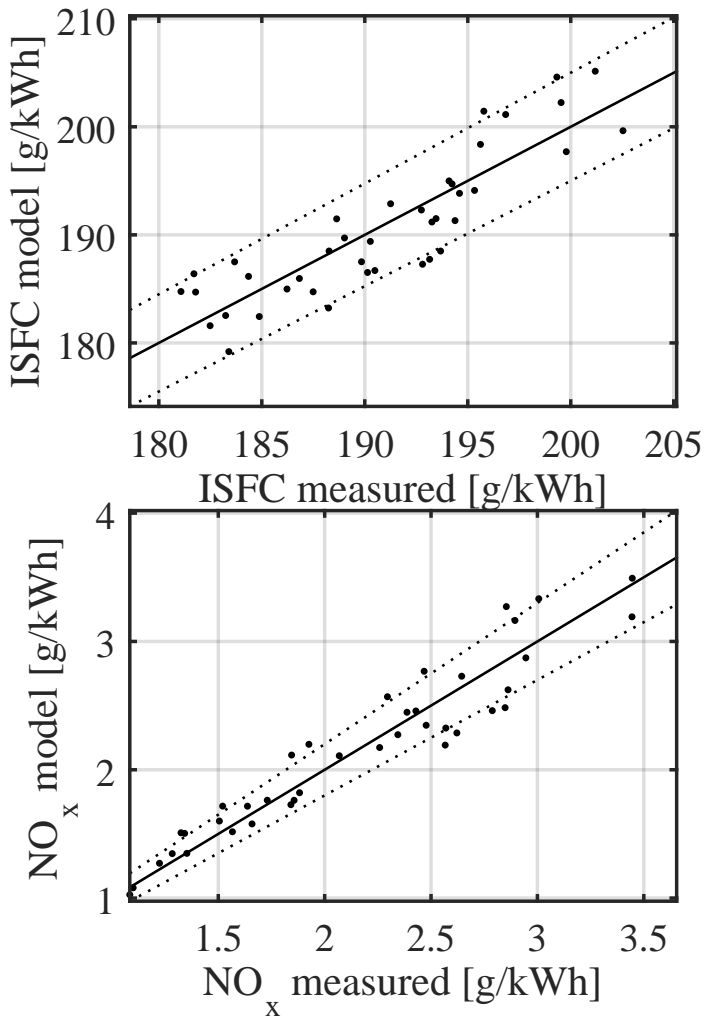

Fig. 1: Comparison between modelled and experimental Indicated Specific Fuel Consumption (ISFC) and $\mathrm{NO}_{\mathbf{x}}$ emissions. Dotted lines correspond to $2.5 \%$ differences in the ISFC and $10 \%$ differences in $\mathrm{NO}_{\mathbf{x}}$.

it is independent of the type of model used to describe these mechanisms. Therefore, it can be concluded that the accuracy of the presented model is reasonably good.

\section{PROBLEM FORMULATION}

The problem examined in the present paper consists in finding the sequence of burning rates that minimises the indicated specific fuel consumption (ISFC) over the engine thermodynamic cycle between the IVC and EVO for an operating point defined as the set of engine speed, fuel injected and cylinder gas conditions at the IVC (temperature, pressure, composition). Given that:

$$
I S F C=\frac{m_{f}}{W_{i}}
$$

as the fuel injection $\left(m_{f}\right)$ is included in the operating point 
definition, minimising the ISFC is equivalent to maximising the indicated work $\left(W_{i}\right)$. For the sake of formulation simplicity, the last option has been chosen. In addition to maximise the function $W_{i}$, the optimal burning rate policy should fulfil the following constraints:

- The maximum cylinder pressure should be below certain limit $\left(p<p^{\max }\right)$ to avoid engine damage.

- The maximum cylinder pressure derivative should be below certain limit $\left(d p<d p^{\max }\right)$ to avoid engine damage and excessive noise emissions.

- The amount of $\mathrm{NO}_{\mathrm{x}}$ emitted should be below certain limit $\left(N O_{x}<N O_{x}^{\max }\right)$. If this constraint is not included, provided the trade-off between emissions and efficiency the optimal solution may tend to a combustion with excessive $\mathrm{NO}_{\mathbf{x}}$ that would make impossible to fulfil with current emission regulations.

To deal with the previous objective, consider the following general dynamic equation of the system:

$$
\frac{\mathrm{d} x}{\mathrm{~d} \alpha}=f(x, u, \alpha)
$$

where the state vector $(x)$ consists of the cylinder pressure $(p)$, heat released $\left(q_{b}\right)$ and the formed $\mathrm{NO}_{\mathbf{x}}\left(x=\left\{p, q_{b}, N O_{x}\right\}\right)$, and $f$ is a generic function containing the model equations (1), (2) and (5) respectively. Meanwhile, the control action (u) is the fuel burning rate as introduced in equation (2). Note that the time dependence has been replaced by an angle $(\alpha)$ dependence provided that the engine speed is considered constant. This modification allows to easily deal with the integration limits of the problem (IVC and EVO) for different operating points without any loss of generality.

Consider the OPC consisting of finding the optimal control policy $\left(u^{*}(\alpha)\right)$ that maximises the following function $(J)$ over a defined horizon $\left(\alpha_{I C V}\right.$ to $\left.\alpha_{E V O}\right)$ :

$$
J=\int_{\alpha_{I V C}}^{\alpha_{E V O}} L(x, u, \alpha) \mathrm{d} \alpha
$$

In the case at hand, the function $J$ represents the work done along the cycle, and therefore the function $L$ is:

$$
L(x, u, \alpha)=W_{i}=p(x, u, \alpha) \frac{\mathrm{d} V}{\mathrm{~d} \alpha}
$$

where the variation of volume with angle is a priori known since piston deformations are neglected. Note that the only state variable affecting the cost function is the pressure and then, $q_{b}$ and $N O_{x}$ are included in the state vector with the aim of introducing the OCP constraints. Particularly, the heat released should be introduced to assure that the fuel burnt during the cycle does not exceed the fuel injected:

$$
\int_{\alpha_{I V C}}^{\alpha_{E V O}} q_{b}(u) \mathrm{d} \alpha \leq m_{f} L H V
$$

or making use of expression (2), in terms of the control variable:

$$
\int_{\alpha_{I V C}}^{\alpha_{E V O}} u(\alpha) \mathrm{d} \alpha \leq 1
$$

where obviously, to maximise the indicated work all the fuel injected should be burnt, so the equal sign prevails.

Analogously, the third state of the problem $\left(N O_{x}\right)$ is included to take into account the constraint on the maximum $\mathrm{NO}_{\mathbf{x}}$ emissions:

$$
\int_{\alpha_{I V C}}^{\alpha_{E V O}} N O_{x}(u, x, \alpha) \mathrm{d} \alpha \leq N O_{x}^{\max }
$$

Note that the optimisation problem consists of one control variable and three states then leading to significant complexity in the solution. In order to simplify the problem consider the Hamiltonian function for the OCP defined by equation (8), subject to the dynamics shown in equation (7) and the constraints in expressions (10) (or (11)) and (12): 


$$
H(x, u, \alpha)=L(x, u, \alpha)+\lambda^{T} f(x, u, \alpha)
$$

where $\lambda$ is a co-state vector $\left(\lambda=\left\{\lambda_{p}, \lambda_{q_{b}}, \lambda_{N O_{x}}\right\}\right)$ whose elements are related to the different states of the problem and allows to introduce the system dynamics in the cost function [15]. Pontryagin's Maximum Principle ensures that for the optimal solution $u^{*}$, the co-state follow:

$$
\frac{\mathrm{d} \lambda^{*}}{\mathrm{~d} \alpha}=-\frac{\partial H}{\partial x}
$$

In the case at hand, since the cost function does not depend on $\mathrm{NO}_{\mathbf{x}}$, then:

$$
\frac{\partial H}{\partial N O x}=0
$$

and then the co-state associated to $\mathrm{NO}_{\mathbf{x}}\left(\lambda_{N O_{x}}\right)$ is constant. Therefore the function $L$ can be modified in order to include the $\mathrm{NO}_{\mathrm{x}}$ constraint as an additional parameter:

$$
L^{\prime}\left(x^{\prime}, u, \alpha\right)=L\left(x^{\prime}, u, \alpha\right)-\lambda_{N O_{x}} N O_{x}\left(x^{\prime}, u, \alpha\right)
$$

where $x^{\prime}$ is the new state vector $\left(x^{\prime}=\left\{p, q_{b}\right\}\right)$ that does not include $\mathrm{NO}_{\mathbf{x}}$. Replacing the cost function (9) by (16) allows to reduce the number of states of the problem, then simplifying its solution but a proper $\lambda_{N O_{x}}$ value should be selected in order to fulfil the $\mathrm{NO}_{\mathbf{x}}$ constraint. Note that $\lambda_{N O_{x}}$ weights the importance of $W_{i}$ and $\mathrm{NO}_{\mathbf{x}}$ emissions in the optimisation function (16). Then, if $\lambda_{N O_{\mathrm{x}}}=0$, the optimisation only considers the $W_{i}$ term, and the optimisation will tend to a control policy minimising the ISFC without considering any penalty in the $\mathrm{NO}_{\mathbf{x}}$ emissions. Conversely, at the limit of $\lambda_{N O_{\mathrm{x}}}$ tending to $\infty$, the $\mathrm{NO}_{\mathbf{x}}$ emissions will be minimised without considering the associated penalty on fuel consumption. Sweeping $\lambda_{N O_{\mathbf{x}}}$ from 0 to arbitrarily high values allows obtaining the Pareto optimum of the OCP, i.e. the minimum fuel consumption depending on the limit on $\mathrm{NO}_{\mathrm{x}}$ emissions.

Despite different OC methods to solve the described problem exist, since the number of states and control variables ( 2 and 1 respectively) is small, the problem is specially well suited for the application of Dynamic Programming (DP) [15], which is based on the Bellman's principle of optimality, that informally states that given an OCP, the optimal control policy that solves an OCP in a subset of the main problem, coincides with the optimal control policy of the complete OCP in the considered subset [16]. Accordingly, the DP method consists in applying a proper discretisation of the problem in both controls and states, and then choosing the set of control actions that minimises the cost-to-go function subject to the set of constraints. The DP solver used in this work is a Matlab based code presented in [17]. The same code has been used previously in [7].

\section{Results}

In this section, the results obtained in the optimisation problem are discussed. For the sake of clarity, the problem constraints are progressively introduced, starting from the HRL optimisation without constraints, then introducing the constraints on the cylinder pressure evolution (maximum pressure and maximum pressure derivative), and finally adding the constraint on the $\mathrm{NO}_{\mathbf{x}}$ emissions.

\section{A. Optimal Heat Release Law without constraints}

Consider the OPC presented in section IV without $\mathrm{NO}_{\mathbf{x}}$ nor pressure restrictions. If additionally the system is considered adiabatic, then the solution is the well known constant volume combustion, that essentially consists of an instantaneous combustion (heat release) at the top dead centre (TDC). From the control policy perspective, the solution of such an OPC is an impulse-like control action, i.e. release all the available heat 
instantaneously (with an infinite rate of heat release) at the TDC. Figure 2 shows the evolution of the previous problem states: $p$ and $q_{b}$. In fact, the bottom part of figure 2 shows the $q_{b}$ normalised with the total heat released. The black line in figure 2 represents the solution provided by the DP algorithm to the OCP without restrictions and considering the adiabatic system previously described.
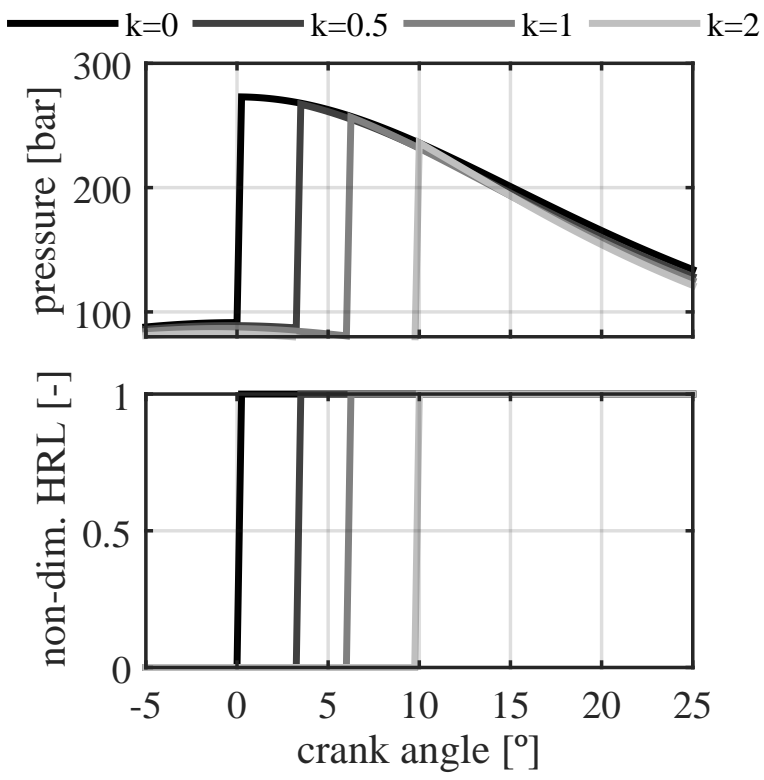

Fig. 2: Effect of the heat transfer on the evolution of the cylinder pressure (top plot) and heat release (bottom plot) without constraints on maximum pressure and maximum pressure derivative. -: $k=0$ (adiabatic); -: $k=0.5 ;-: k=1$ (nominal); - $k=2$.

The obtained solution clearly matches the constant volume combustion then validating the optimisation algorithm used. Note the high values of the in-cylinder pressure (280 bar) and pressure derivative $\left(740 \mathrm{bar} /{ }^{\circ}\right)$ of the optimal solution that would surely damage the engine in a real application. Figure 2 also shows the effect of the heat transfer on the optimal heat release and pressure evolution of the OCP without constraints. The results have been obtained applying a constant factor $k$ to the heat transfer coefficient of equation (3). It is observed that the higher the heat transfer, the latter the optimal combustion appears in order not to reach too high pressures, that, as far as they involve high gas temperatures, lead to important heat losses that jeopardise the engine efficiency. Finally, it can be observed that independently of the considered heat transfer, the optimal control policy involves an instantaneous heat release.

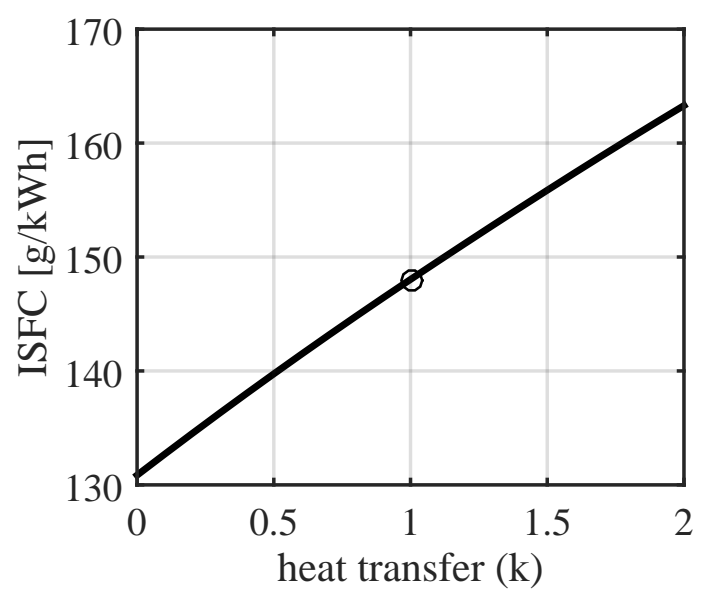

Fig. 3: Effect of the heat transfer coefficient $(k)$ on the minimum Indicated Specific Fuel Consumption (ISFC) without pressure constraints. The circle represents the result for the nominal engine.

The effect of the heat transfer on the minimum ISFC (in the case without constraints) is described in figure 3 , resulting in an almost linear effect of the heat transfer coefficient on the ISFC. The coefficients for the Woschni heat transfer correlations validated by the experimental results in figure 1 lead to a fuel consumption of $148 \mathrm{~g} / \mathrm{kWh}$, that sets a boundary for the minimum ISFC of the actual engine that can be achieved if no constraints are considered. The minimum achievable ISFC by the engine if it were completely adiabatic at the studied operating conditions is $131 \mathrm{~g} / \mathrm{kWh}$. Accordingly, the heat transfer involves a penalty of $17 \mathrm{~g} / \mathrm{kWh}$. Therefore, despite heat transfer consumes around a $30 \%$ of the total energy released during the combustion, the improvement of a completely adiabatic engine will be limited to a $11,5 \%$, even in the case of not considering pressure restrictions. The remaining $18,5 \%$ increases the exhaust gas energy. 

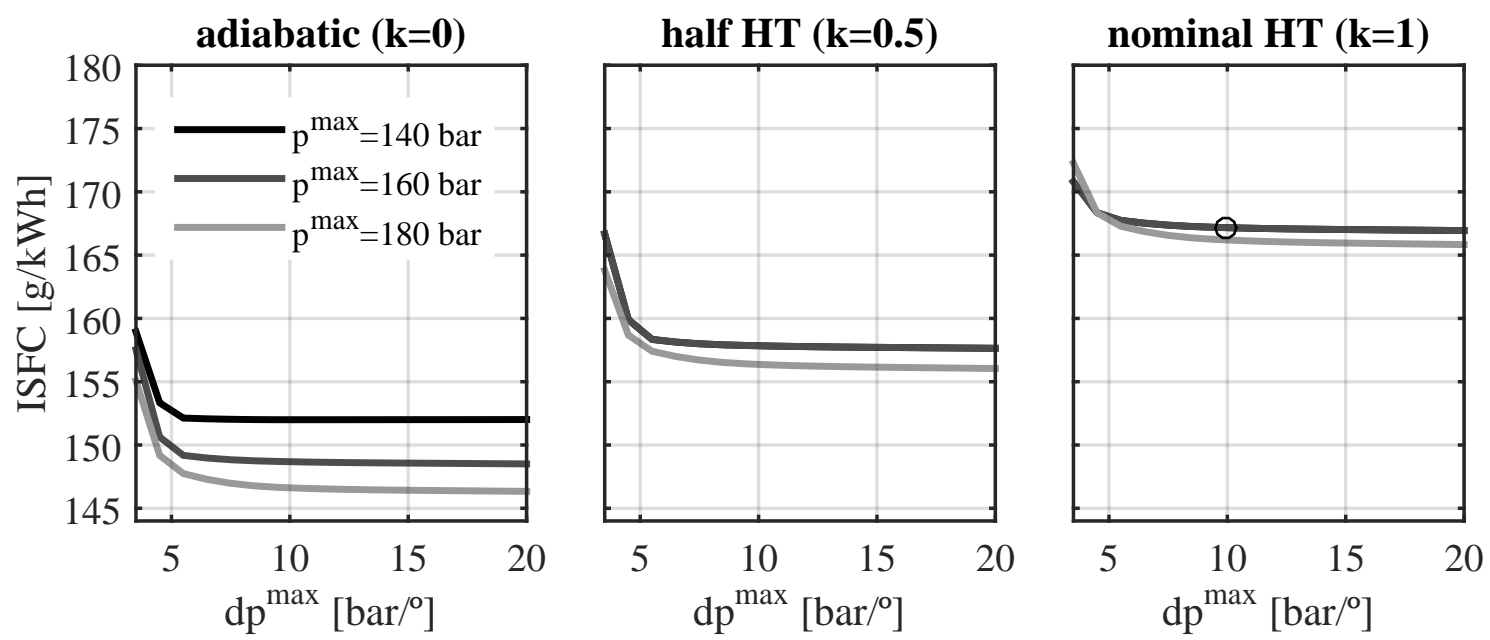

Fig. 4: Effect of the maximum pressure derivative $\left(d p^{\max }\right)$ on the minimum Indicated Specific Fuel Consumption (ISFC) as a function of the limit on maximum cylinder pressure $\left(p^{\max }\right)$ (-: 140 bar; -: 160 bar; -: 180 bar). Left: adiabatic engine. Center: egine with half the heat transfer of the nominal engine. Right: Nominal engine (the circle represents the nominal engine with its actual constraints on maximum pressure and maximum pressure derivative).

\section{B. Optimal Heat Release Law with pressure constraints}

Figure 4 shows the effect of the maximum pressure derivative $\left(d p^{\max }\right)$ and the maximum cylinder pressure $\left(p^{\max }\right)$ on the minimum ISFC for different heat transfer hypothesis. It can be observed how for a given level of heat transfer (e.g. the nominal case) and cylinder pressure limit, the ISFC is progressively reduced as the pressure derivative limit increases. Particularly, the ISFC tends to a minimum that becomes lower as higher maximum cylinder pressures are considered. Increasing the maximum cylinder pressure typically leads to an increase in the area of the $p-V$ diagram, which naturally drives to higher indicated work and consequently to lower ISFC. Also, some kind of asymptotic behaviour is observed since there is a $d p^{\max }$ from which the ISFC is not reduced anymore. This limit in the $d p^{\max }$ which increase leads to lower ISFC appears earlier as the maximum pressure is reduced, not in vane, the lower the maximum cylinder pressure the sooner it can be achieved.

Regarding the effect of the heat transfer on the engine efficiency the results of figure 4 are in line with those presented in section V-A and figure 3: the higher the heat transfer the higher the ISFC. However, figure 4 also shows that the potential of increasing the maximum cylinder pressure as a method to reduce the ISFC is limited by heat transfer effects. In this sense, increasing the maximum cylinder pressure limit from 140 to 180 bar involves a $3.3 \%$ rise in the efficiency of the adiabatic engine, while the same pressure limit increase only leads to a $0.6 \%$ improvement in efficiency with the nominal heat transfer $(k=1)$.

Regarding the impact of the heat transfer on the optimal heat release law (HRL) and the optimal cylinder pressure evolution for the constrained OCP, results in figure 5 show that the higher the heat transfer the later the optimal combustion takes place, which is in line with the results obtained in the OCP without restrictions (figure 2). Again, the reason for such behaviour is that the earlier the combustion, the higher the pressure and temperature in the combustion chamber, so the higher the heat losses. In fact, provided a maximum cylinder pressure of $160 \mathrm{bar}$ and a maximum pressure derivative of $10 \mathrm{bar} /{ }^{\circ}$, the optimal solution is to burn the available fuel in such a way that the maximum allowed pressure derivative is achieved, then, when the maximum allowed pressure is reached the fuel 
burning rate should be reduced to keep constant the cylinder pressure until all the fuel is burnt. No matter the importance of heat transfer (between the limits considered in this study), the optimal policy in the evolution of the pressure and heat release remains the same. The only difference is the start of combustion (SoC), which, as previously stated, should be delayed as the heat transfer increases.
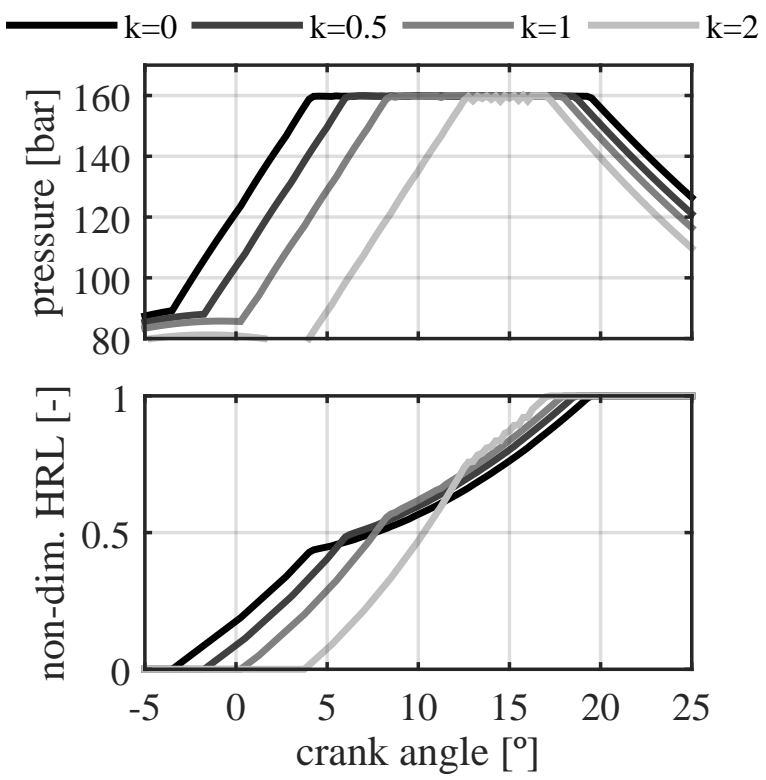

Fig. 5: Effect of the heat transfer on the evolution of the cylinder pressure (top plot) and heat release (bottom plot). -: $k=0$ (adiabatic); -: $k=0.5 ;-: k=1$ (nominal); $k=2$. The actual constraints of the nominal engine in terms of maximum pressure and maximum pressure derivative (160 bar $\& 10 \mathrm{bar}^{\circ}$ ) have been chosen as reference conditions.

Concerning the effect of the pressure derivative on the optimal heat release law (HRL) and cylinder pressure evolution, figure 6 shows that the conditions at the end of the combustion remain independent of the pressure derivative limit imposed, in this case the combustion finishes at $18^{\circ}$ ATDC and reducing the pressure derivative limit involves an earlier start of combustion. In short, figure 6 shows that the optimal combustion process is divided in two main phases. In the first one, the heat release rate is limited by the maximum allowed pressure derivative. The second phase starts once the maximum pressure limit is reached, where the pressure derivative constraint is not active anymore and the maximum heat release rate is limited by the maximum cylinder pressure restriction.
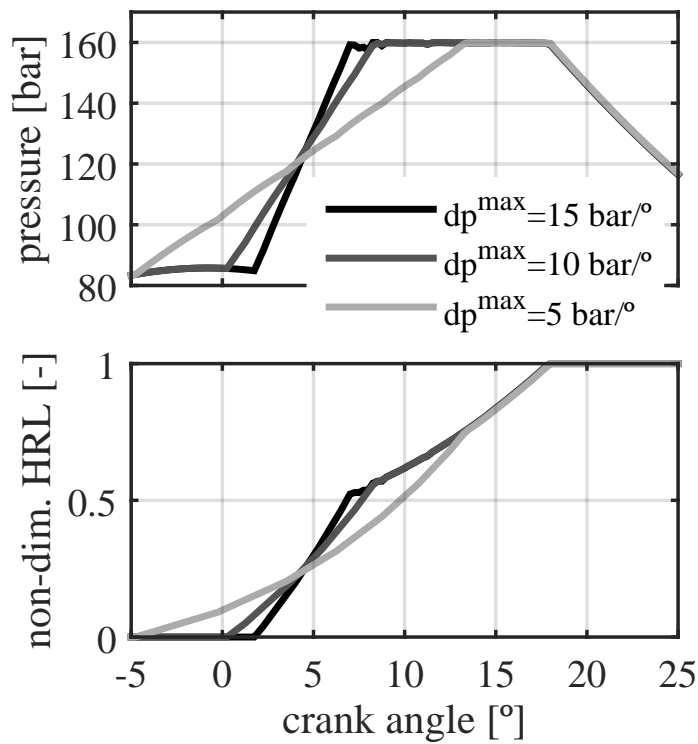

Fig. 6: Effect of the pressure derivative $\left(d p^{\max }\right)$ on the evolution of the cylinder pressure (top plot) and heat release (bottom plot). -: $d p^{\max }=15 \mathrm{bar} /{ }^{\circ} ;-: d p^{\max }=10 \mathrm{bar} /{ }^{\circ}$ (nominal); -: $d p^{\max }=5 \mathrm{bar} /{ }^{\circ}$. The actual constraints of the nominal engine in terms of maximum pressure and heat transfer (160 bar $\& k=1$ ) have been chosen as reference conditions.

Finally, figure 7 shows the effect of the maximum pressure limit at iso-heat transfer and iso-pressure derivative limit on the optimal heat release (HRL) and cylinder pressure evolutions. In this case, the first stages of the combustion process are not sensitive to the maximum pressure constraint, and the pressure derivative limit governs the optimal heat release law. Given a constant pressure derivative, the lower the maximum pressure limit, the sooner it is reached. Then, once the pressure achieves its limit, the rate of heat release is reduced to keep the maximum pressure until the end of the combustion process.

1) Explicit optimal HRL policy: From previous results, one can observe that with independence of the heat transfer, the maximum pressure or pressure derivative limitations, there is a general rule that is always fulfilled: the optimal policy consists of a fast and early HRL limited by the pressure 

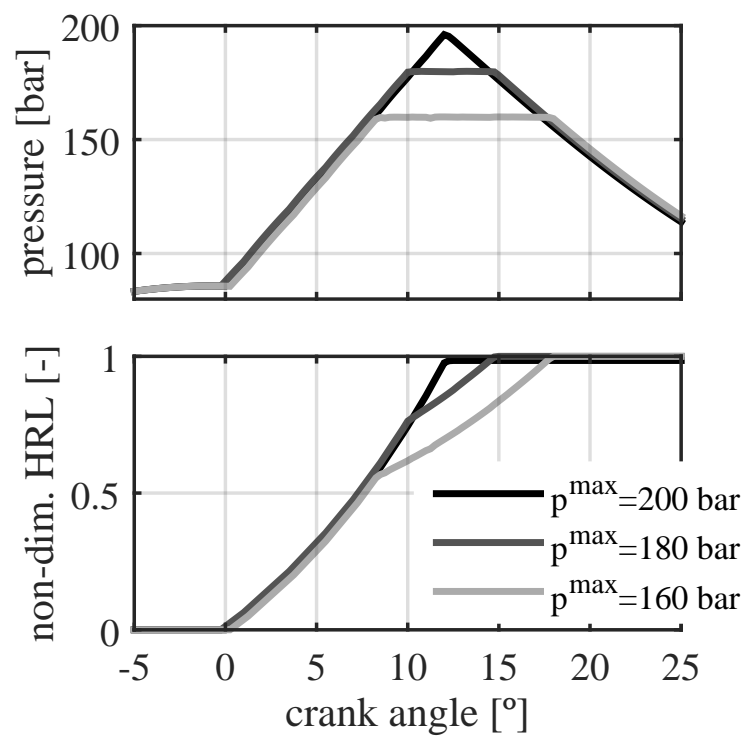

Fig. 7: Effect of the cylinder pressure limit $\left(p^{\max }\right)$ on the evolution of the cylinder pressure (top plot) and heat release (bottom plot). -: $p^{\max }=200$ bar; -: $p^{\max }=180$ bar; -: $p^{\max }=160$ bar (nominal). The actual constraints of the nominal engine in terms of heat transfer and maximum pressure derivative $\left(k=1 \& 10 \mathrm{bar}^{\circ}\right)$ have been chosen as reference conditions.

constraints. In particular, the optimal HRL rate is initially limited by the maximum pressure derivative and once the maximum allowed in-cylinder pressure is reached, the optimal HRL rate is that keeping constant in-cylinder pressure until the fuel is completely burnt. In this sense, the following rule can be identified:

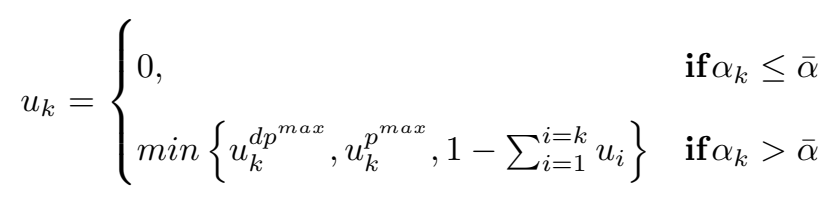

where $u_{k}^{d p^{\max }}$ and $u_{k}^{p^{\max }}$ are the control actions leading to the maximum pressure derivative and maximum cylinder pressure allowed at angle $\alpha_{k}$ respectively. The term $1-\sum_{i=1}^{i=k} u_{i}$ ensures that the maximum released heat does not exceed the fuel energy. Also, $\bar{\alpha}$ is a calibration parameter representing the start of combustion that can be obtained by shooting or any other optimisation method. In this sense, the use of DP can be replaced by using the rule presented in equation (17) with a swept in the variable $\bar{\alpha}$ to choose the value $\bar{\alpha}^{*}$ that minimises the ISFC. Note that the previous rule can be easily implemented since $u_{k}^{d p^{\max }}$ and $u_{k}^{p^{\max }}$ can be obtained solving equation (1) for $\delta q_{b}$ with the proper $d p$. Figure 8 shows the evolution of the ISFC according to the application of equation (17) to a $\bar{\alpha}$ swept from $-15^{\circ}$ to $15^{\circ}$ for the engine nominal conditions $\left(d p^{\max }=10 \mathrm{bar}{ }^{\circ}, p_{\max }=160 \mathrm{bar}\right.$ and $k=1$ ). The obtained results are compared with the result of the DP optimisation in Table III, and a perfect matching can be observed: the optimum start of combustion is $0.5^{\circ}$ and the minimum ISFC is $167 \mathrm{~g} / \mathrm{kWh}$. Despite obtaining near the same results the explicit optimal HRL policy presented has some advantages regarding the DP method: the computation burden is highly reduced ( 2 orders of magnitude despite a swept on $\bar{\alpha}$ has been done instead of a more efficient optimisation algorithm), discretisation in states and control action are not needed, so the accuracy is higher. Table III shows a summary of the optimal results obtained, the discretisation and the computation requirements of both methods.

\begin{tabular}{lll}
\hline & $\mathrm{DP}$ & Explicit policy \\
\hline Angle discretisation & $0.25^{\circ}$ & $0.25^{\circ}$ \\
$u$ discretisation & 200 points & continuous \\
$p$ discretisation & 350 points & continuous \\
$q_{b}$ discretisation & 200 points & continuous \\
Optimal start of combustion & $0.5^{\circ}$ & $0.5^{\circ}$ \\
Optimal ISFC & $167.4 \mathrm{~g} / \mathrm{kWh}$ & $167.2 \mathrm{~g} / \mathrm{kWh}$ \\
CPU time (standard laptop) & $6120 \mathrm{~s}$ & $64 \mathrm{~s}$ \\
\hline
\end{tabular}

TABLE III: Discretisation and performance indices of DP and explicit optimal HRL policy.

\section{Optimal Heat Release Law with $N O_{\mathbf{x}}$ constraints}

The Pareto front in the tradeoff between ISFC and $\mathrm{NO}_{\mathbf{x}}$ emissions obtained by sweeping the $\lambda_{N O_{x}}$ parameter in equation (16) of the OCP is shown in figure 9. The obtained ISFC increases from $167 \mathrm{~g} / \mathrm{kWh}$ when no $\mathrm{NO}_{\mathbf{x}}$ constraints are con- 


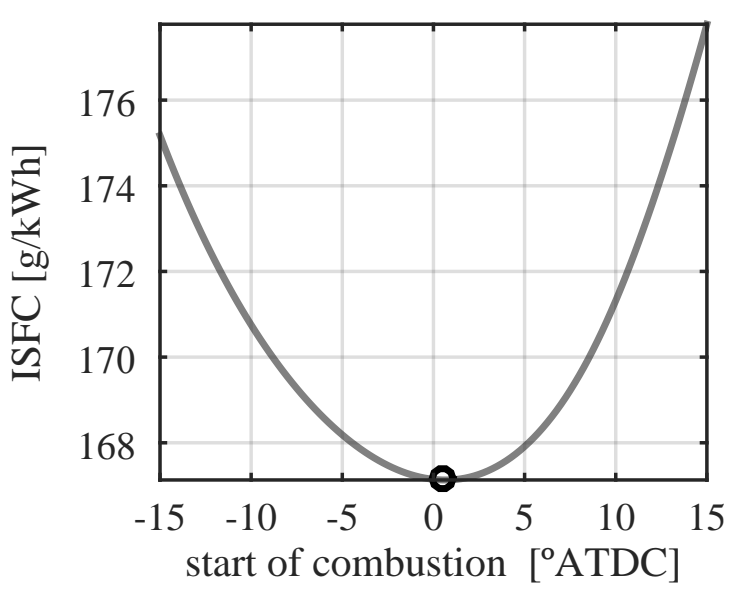

Fig. 8: Swept in the start of combustion parameter of equation (17) (-) compared with the optimal ISFC obtained with DP (O) for the engine nominal conditions $\left(d p^{\max }=10 \mathrm{bar} /{ }^{\circ}\right.$, $p_{\max }=160$ bar and $k=1$ )

sidered $\left(\lambda_{N O_{x}}=0\right)$ to more than $250 \mathrm{~g} / \mathrm{kWh}$ for the maximum values of $\lambda_{N O_{x}}$ tested. Figure 9 points out the negative effect of $\mathrm{NO}_{\mathbf{x}}$ emissions regulations on engine efficiency. In fact, that the tradeoff of figure 9 shows that if $\mathrm{NO}_{\mathrm{x}}$ are not limited the ISFC can be theoretically reduced to $167 \mathrm{~g} / \mathrm{kWh}$ at the expense of high $\mathrm{NO}_{\mathrm{x}}$ emissions around $1.4 \mathrm{~g} / \mathrm{kWh}$. If the $\mathrm{NO}_{\mathrm{x}}$ constraint is set to $0.5 \mathrm{~g} / \mathrm{kWh}$, it can be observed that the minimum ISFC to be attained is $230 \mathrm{~g} / \mathrm{kWh}$. In this sense, a reduction in the $\mathrm{NO}_{\mathrm{x}}$ emissions from 1.4 to $0.5 \mathrm{~g} / \mathrm{kWh}$ has an associated penalty on ISFC of $67 \mathrm{~g} / \mathrm{kWh}$.

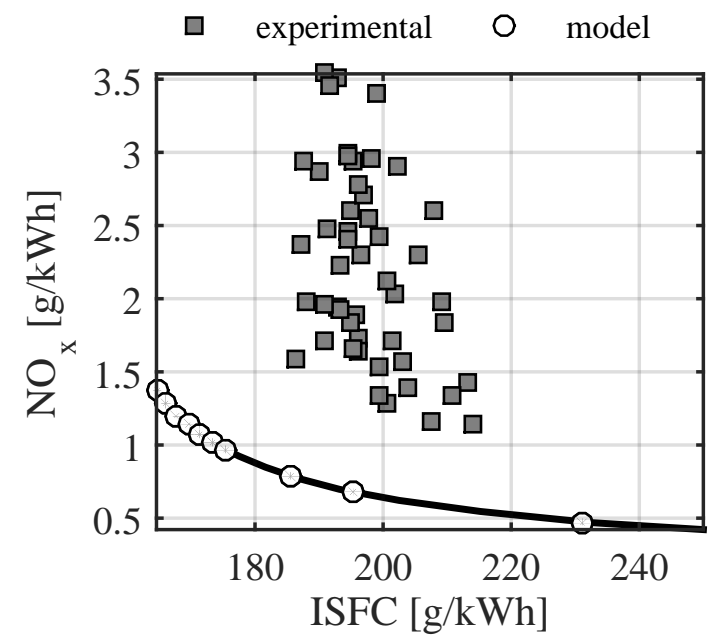

Fig. 9: Tradeoff between ISFC and $\mathrm{NO}_{\mathrm{x}}$ emissions. $\circ:$ Pareto front obtained with DP. $\square$ : experimental results
Figure 10 shows the optimal HRL and cylinder pressure evolutions for different values of $\lambda_{N O_{x}}$ and then different $\mathrm{NO}_{\mathbf{x}}$ emissions according to the colorscale. It can be observed certain pattern in the relation between the $\mathrm{NO}_{\mathrm{x}}$ emissions and the optimal evolution of the OCP states (pressure and fuel burned) from which some clues to shape the optimal combustion rate can be extracted. Starting from the solution without $\mathrm{NO}_{\mathrm{x}}$ constraints $\left(\lambda_{N O_{x}}=0\right)$, one can observe how light reductions in the $\mathrm{NO}_{\mathbf{x}}$ emissions involve a delay in the HRL and therefore the pressure evolution without any change in the pressure derivative. Delaying the HRL law involves a combustion at lower temperatures then with lower $\mathrm{NO}_{\mathrm{x}}$ emissions, see temperature effect in equation (5). If the $\mathrm{NO}_{\mathrm{x}}$ limit is reduced even more, the combustion delay is not enough to satisfy the $\mathrm{NO}_{\mathbf{x}}$ constraint and the rate of HRL should be reduced, see the effect of $q_{b}$ in equation (5). Once an almost constant pressure combustion is reached, additional reductions in the $\mathrm{NO}_{\mathrm{x}}$ emissions are obtained again by means of delaying the combustion process. The relation between the $\mathrm{NO}_{\mathbf{x}}$ emissions and the maximum pressure derivative for the optimal solution of the OCP is depicted in figure 11 . The previous three phases can be clearly identified: the $\mathrm{NO}_{\mathbf{x}}$ emissions can be reduced from $1.4 \mathrm{~g} / \mathrm{kWh}$ to $1.3 \mathrm{~g} / \mathrm{kWh}$ by retarding the combustion keeping maximum allowed pressure derivative. Then, from $1.3 \mathrm{~g} / \mathrm{kWh}$ to $0.95 \mathrm{~g} / \mathrm{kWh}$ the $\mathrm{NO}_{\mathrm{x}}$ emissions can be reduced by means of a reduction of the rate of HRL that involves a reduction of the maximum pressure derivative. Finally, $\mathrm{NO}_{\mathrm{x}}$ emissions below $0.95 \mathrm{~g} / \mathrm{kWh}$ can be only obtained with an almost constant pressure $\left(d p^{\max }=2.3\right.$ bar $/^{\circ}$ ) evolution with each time later combustion.

The experimental results obtained by means of the parametric study described in Table II are also included in figure 9 and 10. For a given $\mathrm{NO}_{\mathbf{x}}$ level, the comparison between the experimental and the optimal ISFC allows the assessment of the engine optimality. One can observe that the experimental 


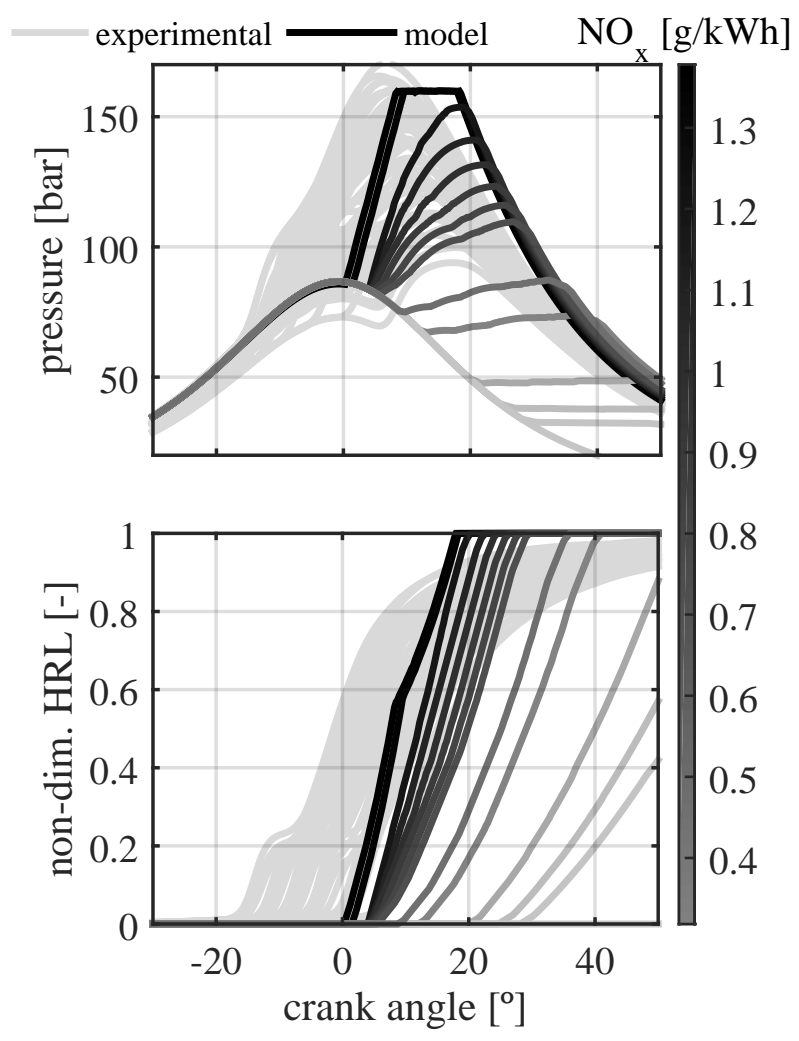

Fig. 10: Evolution of the cylinder pressure (top plot) and heat release (bottom plot). -: DP solution with colorscale representing the $\mathrm{NO}_{\mathbf{x}}$ emissions; -: experimental results

tradeoff is displaced towards higher ISFC and $\mathrm{NO}_{\mathrm{x}}$ emissions, then showing some potential for optimising the engine. It is clear from results in figure 10, that the actual injectioncombustion system is not able to reproduce the calculated optimal HRLs. The optimisation shows that it is necessary to delay the combustion process in order to reduce the $\mathrm{NO}_{\mathbf{x}}$ emissions, which is the general approach followed in current DICI engines, and also in the performed experimental study. The delay in the combustion process in current engines is achieved by delaying the start of injection, by reducing the injection pressure to lengthen the injection process or by introducing inert gases in the combustion chamber through exhaust gas recirculation (EGR). In the present experimental study the first two factors have been modified. Nevertheless, the measures taken in the experimental engine to delay the combustion also involve a noticeable combustion slowdown.

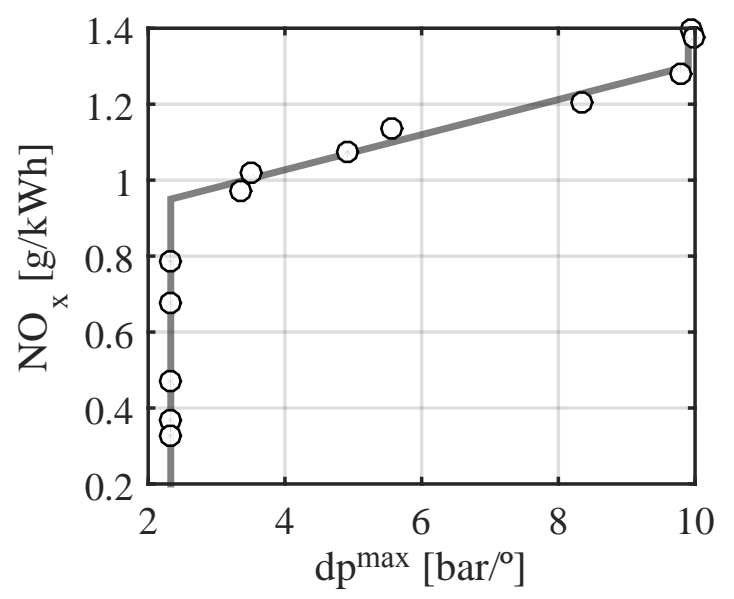

Fig. 11: Relation between the $\mathrm{NO}_{\mathrm{x}}$ emissions and the maximum pressure derivative $d p^{\max }$ in the cycle.

Actually, shifting the combustion towards late phases of the expansion stroke leads to a combustion process at lower temperature and therefore slower. The injection modulation by using different injection events introduces an additional degree of freedom that contributes to the decoupling, to some extent, of both effects. A pilot injection with different timings has been included in the experimental parametric study, however the delayed but still fast combustion obtained in the optimisation process and shown in figure 10 has not been reproduced experimentally.

Despite the optimisation results provide a lower boundary for the ISFC and $\mathrm{NO}_{\mathbf{x}}$ emissions tradeoff of the engine, the considered model uses the HRL as an input, then it is not able to take into account the limitations of the injection system. The application of the OC algorithm to a more physical based model of the combustion process considering the injection pattern as input instead of the HRL will (at least partially) fill the gap between the model based OC and experimental results. In fact, since the injection process has not been taken into account in the performed study, the optimal Pareto front should be taken as a lower limit for the ISFC- $\mathrm{NO}_{\mathbf{x}}$ curve of the engine without injection restrictions. Since the optimal HRL and pressure traces are not achievable experimentally 
it can be concluded that the injection-combustion system has room for improvements. In particular, due to the fact that the engine is extremely small, the required injection split to shape the combustion according to the optimal HRL laws obtained will involve injection quantities below the minimum allowed amounts for the injection system employed.

In addition to the optimisation potential of the current engine, it should be admitted that the $\mathrm{NO}_{\mathrm{x}}$ model is subjected to uncertainties. Despite the $\mathrm{NO}_{\mathbf{x}}$ model parameters were identified to reproduce the experimental measurements provided in figure 1 , the model only considers thermal $\mathrm{NO}_{\mathbf{x}}$ formation, neglecting other $\mathrm{NO}_{\mathbf{x}}$ sources such as the fuel $\mathrm{NO}_{\mathbf{x}}$ formation, prompt $\mathrm{NO}_{\mathrm{x}}$ formation and $\mathrm{NO}_{\mathrm{x}}$ production via $\mathrm{N}_{2} \mathrm{O}$. The differences between the experimental and calculated HRLs that can be observed in figure 10, may lead to nonnegligible differences in the weight of the different $\mathrm{NO}_{\mathbf{x}}$ formation mechanisms involving a model underestimation of the $\mathrm{NO}_{\mathrm{x}}$ emissions.

\section{CONCLUSiOnS}

Optimal Control has been shown as a valid method for shaping the HRL that minimises the ISFC with different constraints, namely: maximum cylinder pressure, maximum cylinder pressure derivative and $\mathrm{NO}_{\mathrm{x}}$ emissions. For that purpose, a validated model of the combustion process including the thermal $\mathrm{NO}_{\mathbf{x}}$ formation has been combined with a DP algorithm. The main contributions of the paper are:

- The use of the Optimal Control framework allows to assess the engine performance with a more representative reference than traditional thermodynamic combustion processes such as constant volume, constant pressure and limited pressure or even arbitrary Wiebe functions. Particularly, the Optimal Control approach provides a basis of comparison that is the HRL providing the best efficiency with some predefined constraints, in the case at hand, maximum cylinder pressure, pressure derivative and $\mathrm{NO}_{\mathrm{x}}$ emissions.

- The OPC solution provides a target HRL to define injection strategies and evaluate the systems or processes with room for improvement. In the case at hand, the analysis has pointed out some limitations in the injection system capabilities to shape the injection and therefore the combustion process.

- The present paper introduces $\mathrm{NO}_{\mathbf{x}}$ constraints in front of previous works where OC was applied to the study of the combustion process that only considered pressure, pressure derivative limitations and knock limitations.

- If the $\mathrm{NO}_{\mathrm{x}}$ constraint is not included, the analysis of the obtained DP solutions has allowed to define an explicit optimal HRL policy, with computation times 2 orders of magnitude below the DP solution and without state and control action discretisation requirements.

\section{NOMENCLATURE}

OD $\ldots \ldots \ldots$ Zero-dimensional

$\alpha \ldots \ldots \ldots$ Crank angle

$B \ldots \ldots \ldots$ Cylinder bore

$\mathrm{CO}_{2} \ldots \ldots$ Carbon dioxide

$c_{m} \ldots \ldots \ldots$ Mean piston velocity

$c_{u} \ldots \ldots \ldots$ Tangential flow velocity

DICI ...... Direct Injection Compression Ignition

DP $\ldots \ldots \ldots$ Dynamic Programming

$d p \ldots \ldots \ldots$ Pressure derivative respect crank angle

$\delta q_{b} \ldots \ldots \ldots$ Heat release rate

$\delta q_{\text {walls }} \ldots$. Heat transfer through cylinder walls

EVO ....... Exhaust Valve Opening

FAR $\ldots . .$. Fuel to air ratio

$\gamma \ldots \ldots \ldots$. Heat capacity ratio

$H \ldots \ldots \ldots$ Hamiltonian function 
HRL Heat Release Law

$h \ldots . .$. Heat transfer coefficient

IVC ....... Intake Valve Closing

ISFC ...... Indicated Specific Fuel Consumption

$J \ldots \ldots \ldots$ Merit function to maximise

$L \ldots \ldots \ldots$ Lagrangian function

LHV ...... Lower Heating Value

$\lambda \ldots \ldots \ldots$ Co-state vector

$m_{f} \ldots \ldots \ldots$ Fuel injected

$\mathrm{NO}_{\mathrm{x}} \ldots \ldots$ Nitrogen oxides

OC $\ldots . . .$. Optimal Control

OCP ...... Optimal Control Problem

$p \quad \ldots \ldots \ldots$ Pressure

$q_{b} \ldots \ldots \ldots$ Heat release

$T \ldots \ldots$ Temperature

$T_{a d} \ldots \ldots$ Adiabatic flame temperature

TDC ...... Top Dead Center

$u \ldots \ldots$. Control action

$V \ldots \ldots$ Volume

$V_{d} \ldots \ldots \ldots$ Cylinder displacement

$v \ldots \ldots \ldots$ Specific volume

$v_{g} \ldots \ldots \ldots$ Gas velocity

$W_{i} \ldots \ldots$ Indicated work

$x \quad \ldots \ldots \ldots$ Control problem state

\section{REFERENCES}

[1] E. Ajav, B. Singh, and T. Bhattacharya, "Thermal balance of a single cylinder diesel engine operating on alternative fuels," Energy conversion and management, vol. 41, no. 14, pp. 1533-1541, 2000.

[2] I. Taymaz, "An experimental study of energy balance in low heat rejection diesel engine," Energy, vol. 31, no. 2, pp. 364-371, 2006.

[3] F. Payri, P. Olmeda, J. Martin, and R. Carreño, "A New Tool to Perform Global Energy Balances in DI Diesel Engines," $S A E$ Int. J. Engines, vol. 7, pp. 43-59, 04 2014. [Online]. Available: http://dx.doi.org/10.4271/2014-01-0665

[4] F. Payri, J. Martin, A. Garcia, and R. Carreño, "Experimental and Theoretical Analysis of the Energy Balance in a DI Diesel Engine," in
SAE Technical Paper. SAE International, 04 2015. [Online]. Available: http://dx.doi.org/10.4271/2015-01-1651

[5] J. A. Caton, "A review of investigations using the second law of thermodynamics to study internal-combustion engines," SAE Technical Paper, Tech. Rep., 2000.

[6] C. Rakopoulos and D. Kyritsis, "Comparative second-law analysis of internal combustion engine operation for methane, methanol, and dodecane fuels," Energy, vol. 26, no. 7, pp. 705-722, 2001.

[7] F. Zurbriggen, T. Ott, C. Onder, and L. Guzzella, "Optimal Control of the Heat Release Rate of an Internal Combustion Engine With Pressure Gradient, Maximum Pressure, and Knock Constraints," Journal of Dynamic Systems, Measurement, and Control, vol. 136, no. 6, p. 061006, 2014.

[8] L. Eriksson and M. Sivertsson, "Computing optimal heat release rates in combustion engines," SAE International Journal of Engines, vol. 8, no. 3, pp. 1069-1079, 2015.

[9] C. Guardiola, J. López, J. Martin, and D. García-Sarmiento, "Semiempirical in-cylinder pressure based model for NOx prediction oriented to control applications," Applied Thermal Engineering, vol. 31, no. 16, pp. 3275-3286, 2011.

[10] M. Lapuerta, O. Armas, and J. Hernández, "Diagnosis of DI Diesel combustion from in-cylinder pressure signal by estimation of mean thermodynamic properties of the gas," Applied Thermal Engineering, vol. 19, no. 5, pp. 513-529, 1999.

[11] G. Woschni, "A universally applicable equation for the instantaneous heat transfer coefficient in the internal combustion engine," SAE Technical paper, Tech. Rep., 1967.

[12] C. Guardiola, B. Pla, D. Blanco-Rodriguez, and P. O. Calendini, "ECUoriented models for NOx prediction. Part 1: a mean value engine model for NOx prediction," Proceedings of the Institution of Mechanical Engineers, Part D: Journal of Automobile Engineering, p. 0954407014550191, 2014.

[13] J. Arrègle, J. J. López, C. Guardiola, and C. Monin, "On board NOx prediction in diesel engines: a physical approach," in Automotive Model Predictive Control. Springer, 2010, pp. 25-36.

[14] F. Payri, J. J. Lopez, B. Pla, and D. G. Bustamante, "Assessing the Limits of Downsizing in Diesel Engines," in SAE Technical Paper. SAE International, 11 2014. [Online]. Available: http://dx.doi.org/10.4271/2014-32-0128

[15] A. E. Bryson and Y.-C. Ho, Applied optimal control: Optimization, estimation, and control. Taylor \& Francis, 1975.

[16] H. P. Geering, Optimal control with engineering applications. Springer, 2007.

[17] O. Sundström and L. Guzzella, "A generic dynamic programming Matlab function," in Control Applications, $(C C A) \&$ Intelligent Control,(ISIC), 2009 IEEE. IEEE, 2009, pp. 1625-1630. 